\title{
UNE MÉMOIRE MÉCONNUE : TÉMOIGNAGES SUR DES ATTAQUES DE 2006 EN ZONE RURALE EN RÉPUBLIQUE CENTRAFRICAINE
}

\author{
Paulette Roulon-Doko \\ LLACAN (CNRS), INALCO, France

\begin{abstract}
L'analyse d'une dizaine de récits de pillage de 2006 survenus dans une zone rurale de l'ouest de la RCA permet de comprendre comment chaque personne a vécu les événements et les réactions individuelles. Ce qui ressort de ces récits est l'expression de la peur, mais aussi une sorte d'audace qui montre la volonté de résistance des peuples et, enfin, la garantie de pouvoir compter sur l'aide de leurs ancêtres. Cette compilation de récits représente la mémoire des événements tels qu'ils se transmettent dans cette culture de tradition orale.
\end{abstract}

MOTS-CLÉS: R.C.A., PILLAGE, 2006, GBAYA, MÉMOIRE, RÉSISTANCE, ANCETRES

Unknown testimonies: remembering the 2006 attacks in rural Central African Republic

Through the analysis of about ten accounts of the 2006 pillaging that took place in a rural area of western CAR, it is possible to understand both how each person experienced the events differently and their individual reactions. What stands out of these accounts is the expression of fear, but also a kind of audacity that shows the people's will to resist, and finally the guarantee that they can rely on the help of their ancestors. This compilation of narratives represents the memory of the events as it is passed on in this culture of oral tradition.

\section{Introduction}

En RCA, il y a eu très peu d'informations sur les exactions commises dans les zones rurales jugées 'pauvres'; ce sont le plus souvent des nouvelles concernant des villes et principalement la capitale Bangui qui sont transmises. J'ai enregistré en 2012, au village de Ndongué ${ }^{1}$, le témoignage d'une dizaine de personnes qui m'ont librement raconté comment elles s'étaient retrouvées face à des bandits qui avaient envahi la région pour la piller au cours de l'année 2006 afin, disaient-ils, de récupérer sur la population des sommes qui leur avaient été promises mais n'avaient pas été réglées. Ces témoignages sont tout d'abord représentatifs de la mémoire de ces événements telle qu'elle est ainsi

1 Je travaille au village de Ndongué depuis 1970 où j'ai régulièrement séjourné jusqu'en 1995 et n'ai pu y revenir qu'en 2011 et 2012, période où la situation politique était alors redevenue 'calme'. 


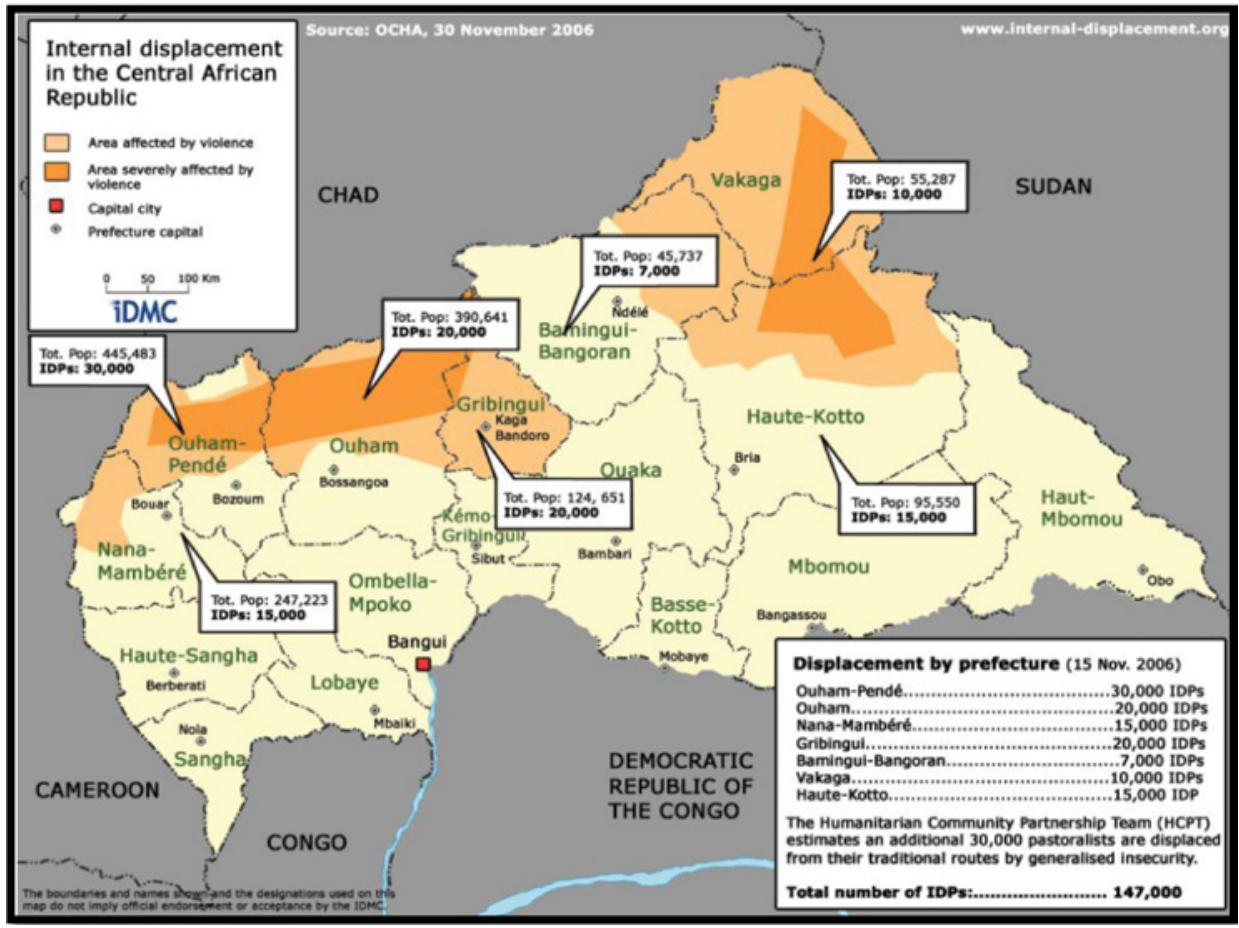

transmise. Ils permettent aussi de saisir tant la perception différente que chacun a de ces événements que la réaction individuelle de chacun. J'analyse en particulier l'expression de la peur, mais aussi une forme d'effronterie qui montre une volonté de résistance, et enfin l'assurance de pouvoir compter sur l'aide de leurs ancêtres.

\section{La situation politique en 2006 en RCA}

En 2006, sous la présidence du Général Bozizé, arrivé au pouvoir suite à un coup d'état en 2003 puis élu en mai 2005, ont eu lieu, dans la commune rurale de Bingué, sous-préfecture de la Nana-Mambéré, des attaques visant les villageois. La situation politique du pays est clairement résumée dans le Rapport de l'IDMC² du 26 janvier 2007 :

Après plus d'une décennie d'instabilité politique par une série de mutineries, de tentatives de coup d'État et de conflits armés, le nombre de personnes déplacées à l'intérieur de leur propre pays aurait triplé au cours de l'année 2006, passant de 50000 en avril à un nombre estimé à 150000 à la fin de l'année. [...] Cette augmentation est en grande partie due à l'insécurité persistante au nord du pays où le gouvernement mène des combats contre plusieurs groupes rebelles. Les bandits de grand chemin (appelés coupeurs de route ou Zaraguinas) et les vols de bétail frappent également le nord, où la présence de l'État comme dans la plus grande partie du reste du pays en dehors de la capitale - est faible voire inexistante.» $[\ldots]$ 
Parmi les rebelles actifs au nord de la RCA, on trouve des combattants fidèles à l'ancien président, Ange Félix Patassé, qui a été renversé par un coup d’État fomenté par François Bozizé, l'actuel président, au pouvoir depuis mars 2003. En outre, certains anciens partisans de Bozizé ont pris les armes contre le gouvernement actuel, estimant qu'ils n'avaient pas obtenu une compensation adéquate pour leur participation au coup d'État. [...]

La plupart des personnes contraintes de quitter leur foyer du fait de l'insécurité provoquée par les combats et le banditisme dans différentes parties du nord ont trouvé refuge dans la brousse, non loin de leurs villages et de leurs champs. Les régions les plus touchées sont les préfectures de Nana-Mambéré, Ouham-Pendé, Ouham, Nana-Gribizi (Gribingui), Bamingui-Bangoran, Haute-Kotto et Vakaga, situées au nord.

\section{Carte des violences en RCA, source OCHA 30 novembre 2006}

Pour la population de Ndongué, village gbaya ${ }^{3}$ de la commune rurale de Bingué, situé à l'extrême sud-ouest de la zone touchée par la violence, on ne peut pas à proprement parler de personnes 'déplacées'. Les Gbaya bodoe sont en effet des chasseurs cueilleurs cultivateurs qui vivent autant dans leur «village» yé que dans la brousse environnante qui représente pour eux le «grand village» gbàyé de leurs ancêtres. Ils connaissent parfaitement ce territoire qu'ils parcourent en permanence pour leurs diverses activités vivrières ou artisanales et sur lequel se situent les terres qu'ils cultivent (manioc, sésame etc.). J'ai pu constater en 2012 que personne au village de Ndongué n'avait quitté le village à la suite des attaques subies en 2006.

L'identité des attaquants est multiple compte tenu de "la présence d'anciennes troupes tchadiennes, des FACA et de la Garde présidentielle, des rebelles centrafricains, des bandits de grand chemin et des rebelles tchadiens" (Ibidem :10-11) attestée alors dans le pays. En 2006 :

Toutefois, l'identité des groupes armés semble connue. Les rebelles comprendraient d'anciens membres des forces de sécurité présidentielles de Patassé ainsi que des combattants démobilisés qui avaient pris part à la rébellion précédente menée par le président actuel. (Ibidem p. 11)

Dans la Nana-Mambéré, les attaques ont tout d'abord visé les Mbororo ${ }^{4}$, des éleveurs peuls nomades dont le bétail constitue une richesse très convoitée. Ceux-ci ont, dès avril 2005, commencé à se réfugier au Cameroun et le processus s'est accéléré en 2006 (Ibidem :12). Cela a conduit les attaquants, à la recherche de richesses mais surtout de nourriture, jusqu'aux villages plus éloignés des routes. C'est le cas de Ndongué qui est situé à $40 \mathrm{~km}$ au sud de la nationale 3, tronçon du corridor Douala-Bangui, qui va de Bouar à

3 Les Gbaya sont une population d'environ un demi-million de personnes qui occupe un territoire situé pour les trois quarts à l'ouest de la République Centrafricaine et pour le dernier quart au centre-est du Cameroun. Le groupe numériquement le plus important appelé Gbaya kara ou Gbaya du Nord est subdivisé en plusieurs sous-groupes dont font partie les Gbaya bodoe chez qui je travaille depuis 1970.

4 Une population qui a longtemps nomadisé dans la région en saison sèche à la recherche de pâturages et avec qui les Gbaya bodoe entretiennent à cette occasion de bons rapports, cf. Roulon-Doko, 2005. 
Garoua-Boulai $(158 \mathrm{~km})$, sur une route très peu fréquentée où ne passent que quelques motos. C'est donc à pied et par la brousse que sont arrivés à Ndongué les attaquants de 2006.

\section{Les attaques de Ndongué en 2006}

La chronologie et les circonstances qui ressortent des témoignages recueillis sont les suivants.

Deux passages au village et des incursions en brousse

Les témoins appellent tous à cette période «l'époque des bandits» ngìmbì kóó rèbêl, sans aucune autre précision de date de type calendaire. Ils avaient déjà entendu parler des rebelles ou des coupeurs de route, mais cela s'était toujours passé loin du village. En dépit des rumeurs qui circulaient parlant de leur présence dans la région et de leurs exactions comme d'un danger imminent «ça sentait le roussi» (PD3) activités habituelles et ont été surpris, soit au village, soit pendant ou au retour d'une de leurs activités en brousse ou dans les champs. L'un des témoins précise d'emblée «qu'il ne voulait pas que son regard se pose sur eux» (GN2) et qu'il projetait de fuir s'ils arrivaient jusqu'ici, tandis qu'un autre, lorsqu'il constate qu'ils ont envahi le village ne peut s'empêcher de manifester une certaine curiosité, tentant de les voir tout en se cachant :

\begin{tabular}{|l|l|l|l|l|l|l|l|l|l|}
\hline mí & káì! & nín & Pó & zàrgínà & nè & hứrrè & Pám & zókwà & hó-ná \\
\hline $1 \mathrm{~S}^{6}$ & INTJ & nom & PL & zarguina & REL & $\begin{array}{l}\text { INAC.se_ } \\
\text { répandre.PROXIMAL }\end{array}$ & 1S & INAC.voir.3P & pas_encore \\
\hline
\end{tabular}

Je me dis 'Merde! Les bandits dont tout le monde parle en ce moment, je ne les ai pas encore vus'. (PD 15)

C'est donc dans un climat d'incertitude et de crainte que survient la première attaque, un matin vers $9 \mathrm{~h}$. Tous ceux qui le peuvent fuient. Les bandits arrivent de Galo. Ils rassemblent, comme ils le font toujours [emploi du répétitif-additif kàá] les hommes qu'ils trouvent sur place, ils les interrogent pour qu'ils dénoncent les «riches» ?ó wàn mbòì (PL/celui/argent) ou, en brousse, leur indiquent un chemin, ils démolissent les portes des maisons, ils récupèrent tout ce qu'ils y trouvent et ils menacent de les tuer sans jamais hésiter à frapper. Un certain nombre d'épisodes se produisent dont certains sont mentionnés par au moins deux témoignages : (i) un Mbororo qui a failli se faire brûler vif dans une maison où il s'était réfugié, (ii) l'impossibilité pour un rebelle de démarrer la moto volée à un villageois, (iii) la collecte de toutes les poules du village pour les manger. Enfin juste avant la nuit, ils repartent en brousse vers la rivière Yèmbá, abandonnant un village où il n'y a plus rien à piller. Quelques jours plus tard, ils reviendront, débarquant en pleine nuit et la violence est alors immédiate. Ils sortent les gens de leur lit, à la recherche d'un commissionnaire qui ne

5 Chaque récit est référencié par les initiales du narrateur suivi du numéro de la phrase dans son récit.

6 Les gloses utilisées pour le mot-à-mot des transcriptions sont : intj = interjection, inac $=$ inaccompli, $\mathrm{pl}=\mathrm{pluriel}$, rel $=$ relatif, $1,2,3=$ personne du pronom suivi de la mention $S$ pour singulier et $\mathrm{P}$ pour pluriel. 
leur aurait pas rapporté le sucre et le riz qu'ils attendaient. En représailles, ils s'en prendront à son frère aîné qu'ils tabasseront. Pendant cette même période, ils ont aussi pour certains sillonné la brousse, menaçant ceux sur qui ils tombaient au hasard de leurs déplacements. Cette attaque du village et de la brousse alentours a donc duré près d'une semaine.

\section{Qui sont les bandits?}

Plusieurs termes sont utilisés, dans les récits, pour parler d'eux. Le premier terme zàrgínà (9 occurrences), écrit en français local «zarguina ou zaraguina», serait d'origine haoussa ${ }^{7}$ et désigne des bandits de grands chemins ou coupeurs de route ${ }^{8}$. Ce phénomène de "grand banditisme" s'est développé à partir des années 1990 dans la zone frontalière entre le nord de la RCA, le Tchad et le Cameroun en particulier chez les Mbororo où, aux actes de pillage et de racket, se sont ajoutés des prises d'otage et des assassinats (Seignobos, 2011). Éloignés d'un grand axe routier et n'étant pas éleveurs de bétail, les Gbaya bodoe n'ont jamais été confrontés à cela sur leur territoire. Le second terme pour désigner les attaquants rèbêl (7 occurrences) vient du français «rebelle». Les deux termes ont en fin de compte en gbaya le même sens général de «bandit, pillard». Deux narrateurs ont recours à l'adjectif dáy «mauvais-méchant» qui introduit un jugement de valeur pour désigner les attaquants comme «des salauds» ?ó dáy béí (les / / personne) (LM20, FP2). L'un d'eux les nomment aussi Pó dáy mò (les / / chose) (LM2) « des saloperies ». Cependant, la façon la plus récurrente pour les désigner est l'emploi des pronoms personnels ${ }^{9}$ de 3ème personne - du pluriel wà «3P» pour désigner le groupe, du singulier Pà̀ «3S $» \mathrm{~S}^{\prime}$ il n'y en a qu'un seul - qui se trouvent systématiquement opposés au ?ám mí «je (1S)» du narrateur ou au Péć «nous (1P)» du groupe dont il fait partie. Les autres personnes qui sont des gens qu'on connaît sont, elles, désignées par leur nom propre ou leur statut («mon beau-père» fôrám, «un Mbororo» mà Mbóróró, etc.).

\section{Les témoignages enregistrés en mai 2012}

\section{Qui sont les narrateurs?}

Il s'agit des récits de huit hommes d'une durée moyenne de 5 minutes et de deux très brefs témoignages de femmes. L'une était en brousse lorsqu'elle a vu ces rebelles frapper son père sur le chemin qui revient des champs. L'autre revenait de la source portant de l'eau lorsqu'elle les a aperçus sur le chemin de transhumance près du village. Ils l'ont attrapée, l'ont fait tomber, lui ont donné des coups de pieds puis l'ont laissée. Elle s'est

7 “«D'après le dictionnaire haoussa de R.C. Abraham, le mot "zargina” signifie cubes of washing blue, à rapprocher de l'arabe littéraire azraq = bleu [le bleu de lessive], qui servait aussi à faire des marques sur le visage des voleurs pris en flagrant délit sur les marchés [...]. Par extension, le mot "zargina" a été utilisé dans le sens de bandit masqué et coupeur de route car le visage de ces derniers était en général soit caché par un turban, soit maquillé de bleu» (C. Arditi, Lettre des savanes, n², juin 2001, p. 8) ", (Seignobos, 2011: note 5 p. 46).

"En Centrafrique, les premières informations concernant ces bandits de grands chemins ont commencé à circuler dans les années 1980, après que des voyageurs aient été arrêtés et dépouillés par ces brigands dans le nord-ouest du pays". (Ankogui-Mpoko \& Co, 2009, consultable en ligne < http://hal.cirad.fr/cirad-00457233 >). En gbaya les pronoms personnels ne marquent pas le genre. 
ensuite enfuie. Ces deux femmes ont découvert plus tard, en rentrant au village, le pillage des maisons. D'une manière générale, tous, et particulièrement les femmes et les enfants se sont enfuis en brousse dès qu'ils l'ont pu à l'arrivée des rebelles et y sont restés cachés jusqu'au retour du calme. FP a d'ailleurs remarqué qu' «ils chassent les femmes pour qu'elles restent un peu loin d'eux» (FP121).

Les huit hommes qui ont témoigné sont tous des villageois de Ndongué mariés et pères de famille. Ils sont de différents lignages. Le village comporte en effet cinq lignages qui, pour certains, sont divisés en plusieurs quartiers, ce qui fait au total douze «quartiers» bèć-gàrà.

\begin{tabular}{|c|c|c|c|}
\hline Lignages $>$ & quartiers & Lignages $>$ & quartiers \\
\hline \multirow[t]{5}{*}{ Gòngòwèn } & gbàyík fiò & 6òdòé-tòrò & bòdòé-tòrò \\
\hline & dòfìò & \multirow[t]{3}{*}{ 6ògòn } & bògòn 1 \\
\hline & kòktòè 1 & & 6ògòn 2 \\
\hline & kj̀ktòè 2 & & bògòn 3 \\
\hline & sòr-mò & \multirow[t]{2}{*}{ bòyè } & 6òyè 1 \\
\hline Gòzôm & 6òzôm & & 6òyè 2 \\
\hline
\end{tabular}

Tableau 1. Les lignages et les quartiers du village de Ndongué

Le tableau 2 récapitule pour chaque narrateur, son quartier, son âge approximatif en 2006, la durée de son récit et l'endroit où il se trouvait lorsqu'il a été confronté aux rebelles.

\begin{tabular}{|l|l|l|r|l|}
\hline Nom $^{10}$ & Lignage-quartier & Age & Durée & A rencontré les rebelles \\
\hline FP & bòdòé-tòrò & 55 ans & $10: 05$ & $\begin{array}{l}\text { Vers 17h, au retour des champs, après } \\
\text { s'être baigné }\end{array}$ \\
\hline AF & bòg’̀n 2 & 35 ans & $4: 02$ & Le matin au village, devant sa maison \\
\hline BM & bòzôm & 60 ans & $5: 30$ & Le matin au village, à côté de sa maison \\
\hline GN & kòktòè & 50 ans & $2: 42$ & Le matin au village \\
\hline LM & bògòn 1 & 35 ans & $6: 34$ & $\begin{array}{l}\text { Vers 17h, au retour des champs, après } \\
\text { s'être baigné (vus de loin) }\end{array}$ \\
\hline PD & gbàyík-fiò & 30 ans & $3: 37$ & $\begin{array}{l}\text { Le matin au village en revenant de la } \\
\text { source (eau pour ses briques) }\end{array}$ \\
\hline AD & bòyè & 65 ans & $9: 38$ & $\begin{array}{l}\text { L'après-midi, en brousse, occupé à rele- } \\
\text { ver ses pièges }\end{array}$ \\
\hline MG & gbàyík-fì̀ & 60 ans & $6: 31$ & $\begin{array}{l}\text { En brousse, là où il cherchait de l'or dans } \\
\text { la rivière Wèì, vers 15h }\end{array}$ \\
\hline
\end{tabular}

Tableau 2. Les auteurs des témoignages recueillis

10 J'ai remplacé le nom de chacun par des initiales pour permettre l'anonymisation du corpus. 
C'est en fonction des activités qui les occupaient ce jour-là que les uns ont été surpris, soit le matin au village, soit en brousse dans l'après-midi ou à leur retour des champs.

Beaucoup de villageois étaient partis aux champs, comme LM ou FP qui, lui, mentionne qu'il était parti «désherber son champ pour y planter des arachides» tórá mò mó-gùn zàánù (FP 4). On est donc au mois de mars, au tout début de la saison des pluies, lorsque chacun retourne sur le champ dont il a récolté le sésame trois mois plus tôt, pour le nettoyer, le débarrasser de ses mauvaises herbes afin d'y planter ensuite des arachides et diverses plantes vivrières. D'autres, comme MG étaient occupés depuis plusieurs jours à trier des graviers au bord de la Wèì à la recherche d'or. Ils venaient de faire tôt le matin un aller-retour pour chercher du manioc au village qu'ils ont quitté peu avant l'arrivée des rebelles, et se sont fait surprendre par eux en fin d'après midi, en plein travail à la rivière. Ce fut également le cas de $\mathrm{AD}$ parti relever ses pièges tôt le matin et surpris « dans l'après-midi» wèsé gìrá ?è (AD 57) en brousse, au moment où il voulait rentrer au village. Seuls étaient au village, quelques hommes retardés dans leur départ pour les champs (AF, GN) ou ceux qui, comme BM, n'étaient pas en état de se déplacer. Ce dernier mentionne qu'ils sont arrivés «vers $9 \mathrm{~h} .{ }^{11}{ }^{1}$ (BM4). C'est également le cas de PD, occupé depuis le matin à faire des allers et retours à la source la plus proche pour rapporter l'eau nécessaire à la confection de ses briques, qui les a vus alors qu'il revenait chargé d'eau.

\section{La brutalité du contact}

Plusieurs témoignent de la soudaineté et de l'effet de surprise du premier contact, même si la menace était latente. Alors qu'il remonte de la source, en rentrant des champs, FP a un pressentiment, «c'était comme si quelque chose me retenait» (FP 23), et poursuit son chemin lorsqu'il les voit :

Je ne savais vraiment pas que des gens étaient arrivés là. [...] A-t-on déjà vu quelqu'un harnaché de cette façon? Pas possible, me dis-je, ces types-là sortent d'où? (FP 28, 32-34)

Il pense à s'enfuir, mais se retrouve encerclé. AF resté au village, aperçoit un «béret rouge» et s'exclame :

Je fais oh! où pourrais-je m'envoler ? Comme ça, si j'avais pu être déjà parti, je n'aurais pas vu une telle chose. (AF14-15)

A la vue des rebelles, tous ceux qui le peuvent s'enfuient, courant d'une façon inhabituelle. Témoin d'une telle course effrénée, PD rapporte l'échange qui s'en suit :

- Êtes-vous ${ }^{12}$ devenu fou ou êtes-vous la proie d'un démon?

- Il me dit 'Merde ! jette l'eau par terre, regarde donc ce sont les bandits'. (PD17-18)

BM s'étonne pareillement de voir un de ses pères courir ainsi :

Nous avons vu en haut du village là-bas le père $M$ - qui courait, il courait vraiment à toute allure. 'Oh! Qu'est-ce qu'il y a ici qui le fait partir?' Nous avons alors entendu dire 'les rebelles, les rebelles, les rebelles'. (BM5-8)

11 Dit en français.

12 Pour une présentation des formes de politesse et du système de vouvoiement en gbaya, cf. Roulon-Doko, 1993. 
C'est donc un vent de panique qui s'abat sur ceux qui, au village comme en brousse, ne peuvent fuir. Ainsi, GN qui ne voulait pas les voir, se fait surprendre au village et devant la menace repousse l'idée de fuir :

Tandis que je cherche à me retourner en pensant 'oh ! je vais courir', il relève son fusil. Aussitôt mes jambes mollissent. Si je détale des deux pieds alors c'en est fini de moi. (GN17-19)

Quant à MG et ceux qui lavent avec lui le gravier dans la rivière pour y chercher de l'or, ils se font surprendre en pleine activité : «nous avons aussitôt sursauté, comme un seul homme» (MG49-50) sans aucun moyen de fuir.

L'impuissance à laquelle les condamne cette invasion massive et brusque leur fait envisager la mort. Ainsi AD qui étant blessé ne peut songer à fuir déclare "s'ils le veulent qu'ils me tuent» (AD33 et 251), et $\mathrm{PD}$ qui, pensant à sa mère restée au village, décide de revenir, «Si c'est la mort, je mourrai tout simplement avec eux, dit-il» (PD23).

\section{L'attitude des bandits}

Les descriptions détaillées de certains récits permettent de comprendre (i) la façon dont les bandits occupent le terrain et (ii) comment ils se comportent.

\section{Un contrôle immédiat de l'espace et des gens}

Les rebelles sont arrivés en grand nombre. LM caché les voit passer alors qu'ils quittent le village «Ils allaient en colonne avec leur sacs chargés qui faisaient du bruit pour aller sur la grande route là-bas. » (LM22). FP précise l'encerclement qui l'a surpris sur le chemin qui revient de la source et il les dénombre même :

Il y en avait d'un côté du chemin. Il y en avait de l'autre côté du chemin. Ils se cachaient, les uns derrière des termitières, les autres derrière des arbres. » (FP 24-27). Ils sont trente qui reviennent de se baigner dans la rivière, il en reste vingt ici... voilà qu'on remonte avec eux, et d'autres sont encore avec les petits Mbororo sur le chemin de transhumance là-bas. (FP 111-113).

Dans le village, ils s'organisent pour en contrôler les issues et tout déplacement.

Un de leurs chefs dit à l'un d'aller fermer la route d'en haut là-bas, à un autre d'aller fermer la route du bas là-bas, et aux autres que leur travail c'est d'aller casser les portes. Puis d'autres sont partis sur la route du bas et d'autres sont partis sur la route du haut. Ils vont étaler les fusils devant leurs chefs sur la route de Galo et sur la route de Kotogon». (FP134-144).

Ensuite ils rassemblent ceux qu'ils trouvent et les questionnent à tour de rôle en les menaçant.

\section{Une manifestation de puissance sans limite}

Les rebelles s'octroient des droits sur tout et tous. Ils donnent des ordres et sont toujours prêts à la violence. Imprévisibles, ils peuvent s'en prendre à n'importe qui, comme à ce «vieil homme» Pó sóká bêm (politesse / vieux / enfant) que l'un frappe à la tête avec un verre (FP126), ou à la mère de PD à qui un rebelle reproche de rester assise immobile 
concluant «qu'elle et son enfant, ils ne lui plaisent pas» (PD47). Toute question devient une menace. Le jeune garçon handicapé qui est resté avec BM au village et l'aide à cuire les poules tuées est ainsi brusquement pris à parti :

Puis D- qui se trouve à côté de moi avait tressé ses cheveux. Voilà qu'ils regardent D- ça alors! et ils me demandent si celui-ci est une femme. Non, leur dis-je, ce n'est pas une femme, c'est donc bien un homme. Alors D- se terre vraiment ici, il a ramené ses jambes en les entrecroisant vraiment très fortement, il poursuit cependant la cuisson pour eux. (BM 85-91)

Ils exigent d'être obéis quoiqu'ils demandent. C'est ainsi qu'ils veulent contraindre GN à grimper dans le manguier pour leur cueillir des mangues, mais deux garçons plus jeunes se présentent d'eux-mêmes pour grimper à sa place (GN20-25). Par contre, il ne pourra pas éviter de «danser» quand un autre, encore «adolescent» mà dáyá ngàì-wíà (un certain / tout jeune / jeune homme) souligne-t-il, le lui ordonne (GN37-40). D'autres offrent une noix de cola à $\mathrm{PD}$, lui interdisant de la fendre ou de croquer dedans pour l'obliger à la mettre entière dans sa bouche au risque de s'étouffer (PD74-76). Ils ne tiennent pas compte des éléments culturels qui leur sont à l'occasion signalés, comme PD qui rechigne à attraper les poules :

Quand je lui dis que ce sont des poules pour la dot. 'Bah ! répond-il, Que je les attrape pour lui, il ne sait pas ce que signifie les poules pour la dot'. (PD49-50) Voyant quelqu'un allongé sur une natte qui leur dit qu'il est malade ${ }^{13}$, ils lui ordonnent de se lever (GN 34-36). Quant aux poules, ils n'en consomment que la chair, négligeant les gésiers, les foies et les pattes qui sont très appréciés des Gbaya (BM74, 79). Ils font également les difficiles avec les mangues ou les avocats qu'ils font cueillir pour les jeter, gaspillant les fruits qu'ils ne trouvent pas à leur goût (GN24-25 et BM 40-42).

Toute initiative est condamnée si elle est remarquée. C'est ainsi que GN qui interpelle Y- à qui parle un rebelle se fait aussitôt reprendre

«Oh !Oh! Je l'appelle, je ne vois pas qu'il est occupé à parler avec lui ?» (GN 42).

Le cas le plus dramatique est celui du jeune frère de MG qui perdra la vie pour s'être assis sur une racine d'arbre.

Comme mon frère G- s'est levé ici, voilà qu'ils lui demandent : 'Et oh! Pourquoi s'assied-il sur une racine d'arbre ? Il n'a pas à s'asseoir sur une racine d'arbre, comme nous qui sommes assis par terre. Alors comment peut-il s'asseoir en hauteur ? Comment se fait il qu'il s'assied en hauteur ? Pour que ce soit bien il doit s'asseoir par terre comme nous'. C'est à ce moment là qu'il lui donne un coup de pied, il lui donne un coup de pied dans le foie, puis il se tourne et saisit la crosse de son fusil dont il en donne à nouveau un coup dans le foie. (MG84-104)

Dans ce climat de menace de mort toujours sous-jacente, les témoignages montrent que la menace est d'autant plus mal vécue qu'elle vise un proche, un parent ou pire un enfant. C'est d'ailleurs ce qui donne à AF la force de s'enfuir sans penser davantage à ce qui pourrait lui arriver.

13 Être allongé sur une natte devant sa maison, au lieu de vaquer à ses activités est la façon normale d'informer que l'on ne se sent pas bien, qu'on est malade. 
Et voilà qu'il dit qu'il va donner un coup de pied à mon enfant qui est assis par terre. Alors je me lève pour prendre l'enfant qu'il est sur le point de frapper et son coup de pied me frappe la bouche, me blessant. Moi je prends alors cet enfant et je détale, c'est ainsi. [...] Moi une fois que j'ai attrapé cet enfant je me suis enfui en revenant jusqu'ici, puis me suis engouffré derrière cette maison là. (AF52-55 60)

D'autant qu'ils n'hésitent pas à tirer sur celui qui cherche à fuir devant eux, comme le raconte LM :

K- voit ce qu'ils font, ce n'est pas supportable. Il s'échappe d'à côté de moi à toute vitesse. Et ils pourchassent K- qui se baisse en passant sous l'avocatier à côté de ma maison, revient en courant sur la route ici. Voilà qu'ils font claquer des balles sur lui bang-bang et ils l'insultent. (LM56-57)

La menace est réelle et le risque toujours là, ce qui n'empêche pas chacun de résister comme il peut.

\section{L'attitude des villageois}

Je présenterai successivement (i) les formes de résistance qui sont opposées aux bandits, (ii) l'expression de la solidarité, (iii) la présence des ancêtres et (iv) les sentiments exprimés.

\section{Les formes de résistance}

Tandis que les rebelles sont vite perdus en brousse et ne savent rien du village, eux qui connaissent parfaitement leur territoire dont ils nomment en brousse chaque rivière, chaque croisement de chemin et au village, chaque arbre, chaque maison, chaque termitière, affichent une ignorance des lieux ou des ressources du village qui relève d'une effronterie qui peut être lourde de conséquences. Chacun lorsqu'il est interrogé sur une direction à prendre en brousse feint l'ignorance.

Au village, les questions portant sur les «riches» ?ó wàn mbj̀i (les / celui / argent) sont souvent accompagnées d'une tentative pour les soudoyer que plusieurs mentionnent. Ainsi PD raconte :

Il me demande alors de lui montrer les places des riches ajoutant que quand il les trouvera et m'en donnera une part. Je réponds : 'Non, voyez, il n’y a aucun riche que je connaisse ici. Les gens, les riches qui vivaient ici, les zarguinas les ont chassés, ils sont tous partis'. (PD35-37)

Cette affirmation de ne rien savoir est récurrente dans les récits. Il en va de même des questions portant sur les possesseurs de vaches. C'est ainsi que FP, ignorant comme il l'explique dans son récit qu'ils avaient réussi à extorquer par des coups à un jeune d'un village proche, la liste des trois propriétaires de vache de Ndongué, affirme à chaque nom cité «d'après une liste écrite en arabe» qu'ils ne sont pas là et se fait brutalement frapper dans le dos avec la crosse du fusil puis tombe à terre. Suit un jeu pervers, où «leur chef intervient pour dire de le ménager, il doit pouvoir répondre sans crainte» puis poursuit avec des questions sur sa famille : 
Est-ce que j'ai des épouses ? Oui je réponds j'ai bien une épouse. Et il continue estce que j'ai des enfants? Je réponds que j'ai quatre enfants. Et dit-il sur ces quatre enfants, il y a combien de filles ? Je réponds : trois filles et un seul garçon. Et il fait 'ah ! Ces enfants là ils peuvent vivre après moi là ?’ D'une saccade je pisse sur moi. Oui, cette parole qu'il vient de dire, merde! ça ne me plait plus du tout. Et il me demande encore si au village on vend des arachides. Je réponds qu'on en vend mais que les vendeurs sont déjà partis aux champs. (FP93-110)

Plusieurs témoignages se font l'écho de ce jeu entre un 'gentil' et un 'méchant' rebelle. C'est ainsi qu'AD décrit longuement l'échange entre les deux rebelles qui l'ont surpris en brousse lorsqu'il relevait ses pièges, l'un voulant les contraindre lui et l'enfant qui l'accompagne à les suivre, l'autre se disant attristé pour lui, ajoutant même :

'Oh ! Est-ce qu'il n'a pas entendu les malheurs que je viens de raconter. S'il a 5000

F.CFA sur lui qu'il me les donne et que je rentre chez moi. (AD164-169)

Il continue cependant à l'interroger sur ses champs, ses récoltes dont $\mathrm{AD}$ prétend que les babouins ont tout mangé, ce qui pousse le 'gentil' à lui demander pourquoi il dort aux champs s'il n'en tire rien, il répond que «Ce n'est pas parce qu'il y a un voleur qu'on doit faire un nouveau champ» (AD185). Et les questions s'arrêtent là.

Lorsque les rebelles perçoivent ce refus de coopérer, ils passent aussitôt aux menaces. Disant ne pas savoir qui vend du tabac dans le village, AF est aussitôt menacé :

Est-ce que je sais qu'il est un rebelle et qu'il ne plaisante pas? Si je fais comme ça il me tuera. Je leur dit que ce que je ne sais pas je le leur dit. (AF23-24)

Contraint de rapporter toutes les poules qu'il voit, BM tente d'en faire échapper certaines :

On ramasse les poules, on les pourchasse, partout. Toi qui est ici ils te poussent 'chasse les poules'. Quant à toi, où qu'aillent les poules, leurs yeux te surveillent tout le temps, te disant 'attrape donc bien ces poules, attrape donc celle-là'. Certaines on les pourchasse comme ça et on pousse cette poule-là sous de grandes broussailles, là où on ne pourra pas l'attraper. Cette poule-là est sauvée. Mais les poules qu'on a poussées pour qu'elles s'enfuient et qui n'ont pas fui on les attrape. (BM58-67)

On voit bien que chacun essaie de résister, en tous cas de limiter les dégâts autant qu'il le peut.

\section{La solidarité}

Chacun lorsqu'il est interrogé sur les maisons du village ou sur les villageois, affirme ne rien savoir ou que l'intéressé est parti, à un enterrement, à l'hôpital, à Berbérati ou Carnot qui sont des villes très éloignées. $\mathrm{AD}$ qui vient de dire qu'il ne sait pas où est le chemin de transhumance précise bien dans son récit:

Le chemin de transhumance est ici-même qui monte vers la maison d'Etienne icimême, je ne le lui indique pas. (AD57) 
Ils utilisent systématiquement le vouvoiement habituel et obligatoire vis-à-vis de leurs aînés (hommes ou femmes des générations antérieures ou aînés de leur génération) et font précéder le terme d'appellation de la marque de politesse ?ó « $\mathrm{POL}$ » : «la mère $\mathrm{X}$ » Pó nàà $X$, «mon beau-père» ?ó fòrám, etc.

Tous partagent le même malheur et sont les victimes de la même menace. Lorsqu'ils apprennent par exemple qu'un jeune d'un village proche a, sous les coups, donné les noms des propriétaires de vaches de Ndongué, comme le mentionne FP pour expliquer, a posteriori, la liste écrite en arabe que sort le bandit qui l'interroge, le ton reste celui de l'énoncé d'un fait, pas d'un reproche. Aucun des témoignages recueillis ne fait état d'une quelconque jalousie qui conduirai à trouver dans ces événements dramatiques l'occasion de régler ses propres comptes. Au contraire c'est bien une solidarité sans faille qui est attestée.

Cette solidarité va d'ailleurs au delà des Gbaya qu'ils soient du village ou non, elle concerne également les Mbororo qui nomadisent régulièrement dans la région et qu'ils connaissent, par leur nom, pour certains. L'un d'eux parle ainsi du groupe de Mbororo capturés comme eux :

Puis ce qu'ils ont fait ici, ils ont rassemblé des Mbororo avec des enfants aussi devant leur 'chef' là-bas juste au croisement. [...] Et eux me poussent devant eux, allons-y jusqu'à ce croisement là-bas, vers leur grand là-bas qui, lui, est assis avec les Mbororo, les Mbororo et leurs enfants. Il a pris des arachides et les a mises devant ces Mbororo pour que les petits Mbororo les mangent. Ils ne disent pas un mot et sont silencieux. (FP49-53, 66-71)

Les cadeaux, comme ces arachides offertes, sont le plus souvent 'empoisonnés', comme le montre la paire de tongues offerte à FP que le bandit lui claque pour finir sur le visage, ou la cola entière avec laquelle PD risque de s'étouffer, sans parler des propositions qui leur sont parfois faites de «partager» ce qui a été pillé dans les maisons (BM 93-97) ou la nourriture qu'ils délaissent, comme les abats de poules, par exemple.

Bien que les brigands parlent le plus souvent en fulfulde, les villageois font bien la différence entre eux et les Mbororo de brousse, également locuteurs de fulfulde, qui sont ici comme eux des victimes. Plusieurs témoignages signalent que des jeunes Mbororos pourchassés en brousse viennent se mêler aux villageois et qu'ils font tous front ensemble. Lorsqu'il leur est demandé des informations sur ces Mbororo, ils ne disent rien et se taisent. L'épisode du jeune Mbororo qui a failli être brûlé vif a d'ailleurs frappé les esprits. Alors trois Mbororo étaient arrivés avec un carton et étaient chez E-. Au moment où ils voient les bandits, deux d'entre eux s'enfuient avec le carton dans la brousse. Le dernier entre dans la maison puis se cache derrière le battant de la porte. Ils mettent une allumette allumée dans la maison et l'un d'eux tend le bout du fusil vers l'intérieur de la maison, attendant ce Mbororo. La maison brûle et le Mbororo reste caché derrière le battant de la porte. Le bandit pense que comme il a attendu sans succès et qu'il n'est pas sorti c'est qu'il a déjà dû s'enfuir avec ses amis. Et il part rejoindre ceux qui cognent sur la porte de la maison du père Abo là-bas. A peine est-il parti que 
le Mbororo sort à toute vitesse de dessous les flammes et part. Un jeune Gbaya et un autre jeune étaient venus eux pour un enterrement à Galo-bokowi, ils avaient fui tous ensemble avec ces Mbororo. Ils étaient allés en brousse puis étaient revenus en courant se mêler à nous. En revenant, il est venu se mettre au milieu de nous. (AF29-59)

\section{L'assurance d'être protégés par les ancêtres}

Les Gbaya vivent sur un territoire qu'ils partagent avec leurs ancêtres qui en brousse sont les maîtres des animaux et sont particulièrement attachés aux insectes et aux oiseaux. Dans les récits, la certitude de pouvoir compter sur les ancêtres est clairement exprimée par LM :

Oh ! Du fait qu'ils ont arrêté certains de ceux-ci ici et si des militaires sont aussi venus, je ne sais plus comment me protéger. Puisqu'ils ne m'ont pas libéré comment pourrais-je m'enfuir ? C'est l'affaire des ancêtres, les ancêtres sont derrière moi. (LM85-87)

Mais le plus souvent ce sont des signes indirects qui attestent de la présence protectrice des ancêtres. Ainsi AD qui vient de faire taire l'enfant qui s'apprêtait à montrer la route qu'il venait de dépasser et à qui le rebelle demande ce qu'ils disent tout bas est sauvé par le passage d'une colonne de fourmis cadavres qu'il désigne en répondant :

Ce sont des fourmis-cadavres qui viennent en colonne par ici dont je parle. Il me dit alors : lève-toi et vient ici. Il saute et les écrase du pied, il saute et les écrase du pied, il les tue, il les tue. (AF81-83)

Les rebelles les libèrent juste après et tandis qu'ils s'enfoncent dans la brousse, il voit «un caméléon qui rampe sur le sol pierreux» puis découvrent «plein de petits oiseaux» sur la rive du ruisseau qu'ils franchissent pour fuir (AF226-232). Tous ces éléments sont interprétés comme le signe de la présence bienveillante et protectrice des ancêtres.

\section{L'expression des sentiments}

La peur provoquée par la situation d'agression subie par les villageois a bien sûr été très forte. Elle est explicitement exprimée par plusieurs. C'est la réaction du corps qui la manifeste d'abord. Ainsi, lorsqu'il comprend que ses enfants sont menacés FP ne peut contrôler la peur qu'il ressent et dit «d'une saccade je pisse sur moi» (FP103), et GN à la vue du fusil pointé sur lui déclare «aussitôt mes jambes mollissent» (GN18).

Enfin au moment où le bandit qui est resté seul avec lui, lui ordonne de s'enfuir, FP s'en va et ressentant toute la vulnérabilité de sa position dit :

La peur m'agite de transes et je repars en courant. Et je me précipite encore une fois dans ces macabos et je vais monter dans ce manguier là-bas. Et comme bien sûr je regarde en arrière. Je vois ce qu'ils font aux choses et je n'en peux plus de peur. (FP182-185) 
Cette peur qui le fait trembler est caractérisée comme la «transe divinatoire» gbáná kii (divination/peur) qui agite le bon sorcier lors de la danse de divination. Et il conclut «je n'en peux plus de peur» kì gàná ngài litt. 'la peur dépasse la force'.

Du côté des bandits, plusieurs témoignages mentionnent des manifestations de joie démesurée lorsqu'ils trouvent quelque chose qui leur plaît.

«Alors des cris de joie résonnent très fort. Ils s'étreignent. Ils poussent des youyous». (FP155-156).

\section{Conclusion}

Sur un plan formel, ces récits suivent globalement le fil temporel de la suite des événements vécus, avec cependant certaines remarques qui apportent une information qu'ils n'ont pu avoir qu'ensuite - la liste écrite en arabe, l'identité de celui qui avait fui en abandonnant ses chaussures, l'envahissement du village juste après leur départ pour leur recherche d'or -, et des indications sur les émotions ressenties pour certains. Témoignant d'une situation de violence et de pillage, la parole exprimée reste maîtrisée, sous contrôle, ce qui lui donne une grande force. Elle informe sur ce qui s'est passé, exprimant la souffrance et la résistance d'une population rurale dont on n'entend jamais la voix. La brutalité des bandits, leur violence gratuite, la menace de mort qu'ils représentent pour tous est donnée à voir, à entendre, mais elle est rarement exprimée en termes de jugement. Seul l'adjectif dán «mauvais-méchant» est utilisé dans deux récits, tout d'abord par FP pour désigner les bandits (?ó dáy béí, ?ó dáy mò), puis par MG qui conclut le récit de la mort de son jeune frère par ce constat :

\begin{tabular}{|c|c|c|c|c|c|c|c|c|c|}
\hline$w \grave{a}$ & $d \grave{\varepsilon} \dot{\varepsilon}$ & $n \varepsilon ́$ & ż̀m & gásá & dáy & $m \grave{~}$ & $P a ́$ & $s \check{n}$ & hï-hï \\
\hline${ }_{32} \mathrm{P}$ & ACC.faire.D & FOC & AUGMENTATIF & grand & mauvais & chose & ÊTRE-LOC & dans.D & là-m \\
\hline
\end{tabular}

C'est un acte criminel qu'ils ont fait à ce moment-là. (litt. très grosse mauvaise chose) (MG180)

Ces récits nous apprennent aussi la grande force du lien familial particulièrement souligné par le respect des formes habituelles de politesse (marque de politesse sur les noms, vouvoiement des aînés), mais attestent aussi de l'existence d'un lien entre villageois au sens large qui s'étend même aux nomades mbororo qu'ils connaissent, lorsque ceux-ci sont pris dans la même tourmente qu'eux. A la brutalité des agresseurs répond une solidarité sans faille. Chacun, à son niveau, tente de résister, de s'opposer lorsqu'il le peut, en taisant ce qu'il sait et en ne dénonçant personne. Enfin ces récits sont bien inscrits dans un espace que tous connaissent et pratiquent et dont ils savent reconnaître les signes qui montrent la protection que leurs ancêtres leur apportent.

La mémoire collective du groupe se nourrit de ce type de récits qui transmettent une information qui permet à chacun de chercher comment mieux se protéger et d'être 'averti' pour l'avenir. 


\section{Bibliographie}

IDMC (internal displacement monitoring center-Norwegian refugee council) Déplacements internes en République centrafricaine : une crise de protection, le 26 janvier 2007.

http://www.internal-displacement.org/assets/publications/2007/200701-af-car-internaldisplacement-in-central-african-republic-country-fr.pdf

Roulon-Doko, Paulette, 2005, «Mon ami mbororo, 20 ans de contact Gbaya-Mbororo, 1970-1990», in Baumgardt U. et Derive (dir.) Paroles nomades, écrits d'ethnolinguistique africaine, Paris, Karthala, pp. 281-287.

Roulon-Doko, Paulette, 1993, «Les personnels et les modalités de vouvoiement en gbaya 'bodoe (Centrafrique)», Linguistique Africaine, $\mathrm{n}^{\circ} 11$, Paris, pp. 67-81.

Seignobos, Christian, Le phénomène zargina dans le nord du Cameroun Coupeurs de route et prises d'otages, la crise des sociétés pastorales mbororo, Afrique contemporaine, 2011/3 ( $\left.{ }^{\circ} 239\right)$, pp. 35-59.

Ankogui-Mpoko, Guy-Florent, Kedeu Passingring, Boniface Ganota, David Kadekoy-Tigague, Insécurité, mobilité et migration des éleveurs dans les savanes d'Afrique centrale, in L. SeinyBoukar, P. Boumard, Savanes africaines en développement : innover pour durer, Apr 2009, Garoua, Cameroon, Cirad, 10 p. $<$ http://hal.cirad.fr/cirad-00457233 > . 


\section{Annexe : résumés des récits du corpus}

\section{Récit de FP}

${ }^{1}$ De retour des champs, la baignade et le départ des femmes. Il reste seul, se baigne et remonte. ${ }^{2} \mathrm{Il}$ a un mauvais pressentiment. ${ }^{3} \mathrm{Il}$ y a des rebelles partout. ${ }^{4} \mathrm{Il}$ s'étonne de leur tenue. ${ }^{4}$ Incrédule, ${ }^{5}$ il pense à fuir mais se retrouve encerclé. Suit alors une série de questions ${ }^{6}$ sur ses activités, ${ }^{7}$ sur le village, ${ }^{8}$ sur les propriétaires de vaches, tandis qu'il découvre que des ${ }^{9}$ Mbororo ont également été capturés. Celui qui l'interroge doute de ses réponses. ${ }^{10} \mathrm{Il}$ voit qu'ils ont donné des arachides aux enfants mbororo. L'interrogatoire continue sur ${ }^{11}$ où est l'aîné de D- tandis qu'il ${ }^{12}$ sort un papier écrit en arabe. Il apprendra plus tard qu'il s'agit d'une liste extorquée sous la menace à quelqu'un d'un village voisin. Il lui repose la même ${ }^{11}$ 'question, et il lui répond qu'il est parti à Berbérati. Il pose ensuite des questions sur ${ }^{13}$ les trois propriétaires de vaches du village, il répond qu'ils ne sont plus là. ${ }^{14} \mathrm{Il}$ se fait alors frapper avec la crosse du fusil et tombe. ${ }^{14}$ Le chef dit de ne pas lui faire du mal qu'il puisse répondre. Il se relève et les questions reprennent sur ${ }^{15}$ sa famille (femme et enfants), avec ${ }^{16}$ une menace indirecte de le tuer. ${ }^{17} \mathrm{Il}$ pisse sur lui de peur. Aux questions pour savoir ${ }^{18}$ où acheter des arachides, il dit que les vendeurs sont aux champs. ${ }^{19}$ Il décrit le bain des rebelles puis les dénombre tandis qu'ils remontent sur le village. ${ }^{20}$ Ils tombent sur la femme de M-, la chassent en avant [ils ne veulent pas être à côté de femmes]. ${ }^{21}$ Rencontrant un vieux, il le frappe d'un coup de verre dans la tête. Nouvelle question sur ${ }^{22}$ où est sa maison, la lui désigne. ${ }^{23} \mathrm{Il}$ inspecte sa maison puis ${ }^{24}$ distribue les tâches : fermer les accès au village, casser toutes les portes, étaler les fusils par terre. Il se retrouve assis devant une maison avec le chef et son adjoint. Mais ${ }^{25}$ des hurlements de joie devant une maison au loin, attire le chef qui y va... Lui n'a aucune idée de ce qu'ils ont pu trouver. ${ }^{26}$ Resté seul avec l'autre, il lui offre une paire de chaussures offerte en compensation des coups reçus et lui donne l'ordre de fuir, ${ }^{27}$ le menaçant de ce qui pourrait lui arriver sinon. Le frappe avec la paire de tongues qu'il lui donne. ${ }^{28} \mathrm{Il}$ décrit sa fuite. ${ }^{29} \mathrm{Il}$ est mort de peur. ${ }^{30}$ Se met à l'abri dans un arbre et regarde de loin les rebelles rassembler leurs prises et partir pour la brousse au Sud.

\section{Récit de $A D$}

${ }^{1}$ Il explique qu'il ne les a pas vu arriver, mais a vu un homme de Galo dans une fuite éperdue. N'ayant pu le rattraper, il décide de retourner se coucher. ${ }^{2}$ Il réfléchit, se traite d'idiot et se dit qu'il ferait mieux d'aller voir ce qui se passe. De toutes les façons, il sort d'une opération au ventre et ne peut pas courir vite. Il restera donc au village. ${ }^{3}$ Le lendemain il part avec un enfant relever ses pièges en brousse. ${ }^{4}$ Or les rebelles avaient installé leur tente sur un terrain pierreux non loin. ${ }^{3}$ Ils visitent leurs pièges, attrapent un rat de Gambie et un petit aulacode. ${ }^{5}$ Puis s'apprêtent à rentrer, lorsqu'ils tombent sur deux bandits qui cherchaient la route de Mèrè. ${ }^{6}$ Contraints à s'asseoir, ils doivent répondre sur ${ }^{7}$ comment aller à Mèrè. ${ }^{8} \mathrm{Il}$ perçoit une situation dangereuse et répond qu'il ne sait pas. ${ }^{9}$ Interrogés sur ce qu'ils transportent, il leur montre ses prises. Questionné sur ${ }^{10}$ pourquoi l'enfant ne comprend pas le fulfuldé, il explique qu'il a vécu à Carnot et vient juste de revenir. ${ }^{11} \mathrm{Il}$ 
se sent piégé. D'autres questions ${ }^{12}$ sur sa famille, ${ }^{13}$ sur le temps pour aller à Carnot. ${ }^{14} \mathrm{Il}$ prétend alors ne pas bien comprendre le fulfuldé. ${ }^{15}$ Mais l'autre le prend mal et il fait profil bas. Les questions reprennent demandant ${ }^{16}$ où est la route d'Abba, ${ }^{17}$ où est le chemin de la maison d'E-. ${ }^{18} \mathrm{Il}$ dit ne rien connaître, il ne peut plus se déplacer depuis plusieurs années. ${ }^{18}$ ' Il bluffe et se vante de ne pas avoir montré le chemin de transhumance. ${ }^{19}$ Les bandits discutent ensuite entre eux, l'un voulant les embarquer avec lui, l'autre, le chef refusant car sa situation l'attriste, dit-il. ${ }^{19}$ ' Ne voudrait-il pas lui donner, s'il les a, 5000 F.CFA. ? L'autre dit qu'il se contentera de lui laisser la vie. D'autres questions encore ${ }^{20}$ sur où il vit et ${ }^{21}$ sur ce qu'il a planté dans son champ. ${ }^{22} \mathrm{Il}$ assure que les babouins ont tout dévoré. ${ }^{23}$ Lorsqu'on lui demande pourquoi il reste quand même aux champs, ce n'est pas parce qu'on vole tes produits que tu abandonnes ton champ pour un nouveau, répond-il. ${ }^{24} \mathrm{Ils}$ se résolvent à lui laisser la vie sauve et à le laisser repartir. ${ }^{25} \mathrm{~L}$ 'enfant s'adresse alors à son père pour lui faire remarquer qu'ils viennent de dépasser la route... Il le fait taire, lui désignant ceux qui les menacent. ${ }^{26}$ Les bandits lui demande aussitôt ce qu'ils disent à voix basse, ${ }^{27} \mathrm{il} \mathrm{montre} \mathrm{alors} \mathrm{une} \mathrm{colonne} \mathrm{de} \mathrm{fourmis-cadavres.} \mathrm{Ils} \mathrm{se} \mathrm{lèvent,} \mathrm{tapent} \mathrm{des}$ pieds, leur disent de s'éloigner. ${ }^{28}$ Le chef donne alors à l'enfant une sacoche et nous dit de filer. ${ }^{29}$ En descendant la colline nous voyons un caméléon sur le sol pierreux et au bord du ruisseau, il y a plein d'oiseaux. ${ }^{30} \mathrm{D}$ 'un bond nous traversons le ruisseau et à l'embranchement, nous passons par les herbes du Laos pour dissimuler notre passage, au cas où ils nous poursuivraient, et regagnons les champs. Voilà ce que j'ai vu. ${ }^{31}$ Mon frère aîné m'a reproché de ne pas avoir fui le village. Mais qu'est-ce-que j'avais qui puisse être pris ? Il pouvait seulement me tuer. Ma femme et mes enfants étaient cachés. Et puis ils cherchaient des riches

\section{Récit de AF}

${ }^{1}$ A l'époque de ces rebelles, c'est à cause d'un enfant que j'ai été impliqué, sinon ils ne m'auraient pas attrapé. Avant de partir aux champs, j'avais préparé des macabos pour mon enfant, mais celui-ci ne voulait pas manger. Et les choses en restaient là. ${ }^{2}$ Comme je regarde vers le haut du village, il y a un béret qui fait une tâche rouge là-bas. ${ }^{3} \mathrm{Je}$ fais oh! où pourrais-je m'envoler ? ${ }^{1} \mathrm{C}^{\prime}$ 'est comme ça que si j'étais déjà parti je n'aurais pas vu une telle chose. ${ }^{4}$ Une fois qu'ils sont arrivés, comme partout ils ont appelé tous les hommes et nous ont rassemblés. Ils demandent ${ }^{5}$ qui vend du tabac dans ce village-ci ? ${ }^{6}$ Je réponds que je ne sais pas. ${ }^{7}$ L'autre le menace : «Est-ce que je sais qu'il est un rebelle et qu'il ne plaisante pas ? Si je fais comme ça il me tuera». ${ }^{6}$ ' Je leur dis que ce que je ne sais pas je le leur dit. ${ }^{8}$ Ensuite ils descendent et arrivent chez A- et commencent à détruire sa maison. ${ }^{9}$ Alors trois Mbororo les ayant vus, s'enfuient. L'un rentre dans une maison à laquelle un rebelle met le feu, restant devant la porte avec son fusil. La maison brûle et le rebelle impatient d'aller voir plus loin ce qui se passe, s'en va. Le Mbororo jaillit alors de la maison et s'enfuit. ${ }^{10} \mathrm{Ces}$ jeunes gbaya et mbororo qui fuyaient la brousse étaient venus se mêler à nous. Tous ont été rassemblés sous le manguier chez la grand-mère N-. ${ }^{11}$ Voilà que je suis sur le point de fuir et mon enfant m'appelle, ils m'ordonnent aussitôt de rester là. ${ }^{12}$ Ils nous ont rassemblés, il y a beaucoup de gens et des savons, du riz, toutes sortes de 
choses sont répandues éparpillées par terre. ${ }^{13}$ Et voilà qu'il dit qu'il va donner un coup de pied à mon enfant qui est par terre. ${ }^{14} \mathrm{Je}$ me lève pour prendre l'enfant qu'il est sur le point de frapper et son coup de pied me blesse à la bouche. Moi je prends alors cet enfant et je détale. ${ }^{15}$ Ensuite ils ont frappé le grand $\mathrm{S}$ - avec un bâton et un maître d'école qui enseigne à Galo-Bokowi. ${ }^{16}$ Moi une fois que j'ai attrapé cet enfant, je me suis enfui et je reviens jusqu'ici et je me suis engouffré derrière cette maison là. ${ }^{17} \mathrm{D}$ 'autres ils sont descendus pour aller à Kotogon là-bas. Ils sont allés attraper le grand G- à Kotogon là-bas et l'oblige à pousser un rebelle assis sur le vélo, ses deux mains sur sa kalachnikoff. Voyez la sueur sur son corps. C'est ainsi qu'ils rencontrent B-. Ils mangent les poules en bas là-bas. Ce que j'ai vu de mes propres yeux c'est ça.

\section{Récit de LM}

${ }^{1}$ A l'époque où les mauvais sont venus ils ne sont pas venus une seule fois. ${ }^{2}$ Tout d'abord c'était quand je revenais du champ, en fin d'après midi et que je remontais vers le village. ${ }^{3} \mathrm{~J}$ 'ai remarqué de très nombreuses marques de chaussures d'homme qui m'ont étonné et j'étais contrarié. Puis encore plus avant. Je trouve alors deux 'souplesses' qui sont là sur cette route. Je fais $\mathrm{Oh}$ ! On a poursuivi quelqu'un sur cette route et il a abandonné ses chaussures sur cette route. ${ }^{4} \mathrm{Il}$ apprendra ensuite qu'il s'agissait des tongues de L- perdues lors de sa fuite. ${ }^{5}$ Alors j'entendis quelque chose qui résonnait au village là-bas. ${ }^{6} \mathrm{Je}$ fais oh! Tiens, ce sont les salauds! ${ }^{7}$ Ils s'en allaient en colonne avec leur sacs chargés qui faisaient du bruit pour aller sur la grande route là-bas après être venus abîmer complètement ce village. ${ }^{8} \mathrm{Il}$ découvre ensuite tout le pillage dans le village et tout ce qu'ils avaient abandonné qu'ils avaient bien sûr laissé restait ici, restait ici. ${ }^{9}$ Deux jours après on a entendu dire qu'ils avaient envoyé un villageois acheter du riz et du sucre pour eux qui devait ensuite le leur apporter. ${ }^{10}$ Or celui-ci a été poursuivi par les gendarmes puis attrapé. ${ }^{11}$ L'ayant attendu en vain, ils avaient trop faim et se sont donc mis en route à la nuit profonde, vers minuit, et ils sont venus au village. ${ }^{12} \mathrm{Ils}$ sont entrés dans les maisons, sortant les gens de leur lit. Ils m'ont maltraité, ils avaient un long fouet en cuir et ils m'ont frappé la nuque, m'ordonnant pour finir de sortir. Suit une série de questions pour avoir ${ }^{13}$ une torche (la leur n'éclairait presque pas), ${ }^{14} \mathrm{du}$ pétrole, ${ }^{15} \mathrm{du}$ manioc, ${ }^{16} \mathrm{des}$ feuilles à gluant. Ils n'avaient pas de torche non plus. ${ }^{17} \mathrm{Il}$ répond à chaque fois qu'il n'y en a pas. ${ }^{18}$ Ils pénètrent chez K-, le tirent dehors, l'emmènent à son tour à côté de moi. Ils attrapent sa femme aussi. ${ }^{19}$ Puis K- ne supportant pas la situation s'échappe d'à côté de moi à toute vitesse. Et ils le pourchassent, font claquer des balles sur lui et l'insultent. Ils reviennent ensuite, ${ }^{20} \mathrm{j}$ 'ai bien pensé à fuir de même, mais cela serait retombé sur sa femme à qui ils font transporter tout ce qu'ils trouvent à manger dans la maison. ${ }^{21}$ Puis ils nous poussent plus loin et nous restons assis par terre. Il y a un gardien. L'un d'eux nous surveille en tenant un fusil. ${ }^{22}$ Puis ceux qui détruisent les portes progressent en les cassant. ${ }^{23}$ Ils ont attrapé A-, sa femme le défend. ${ }^{24}$ Ils ont attrapé beaucoup de personnes qui restent là. ${ }^{25}$ Lorsque j'entends que de ce côté de la route ils ont attrapé A- qui se met à cirer dans leur langue [le fulfulde] 'ya pas de sucre! ya pas de sucre!' Alors ils lui pincent l'oreille avec une pince pour la couper. Il hurle. Puis ils nous mettent à part d'un côté et ils vont 
se retrouver en avant. ${ }^{26}$ Ils ont plaqué à terre O-, le frère aîné du villageois envoyé en commission. Ils le tapent. Ils cognent la crosse du fusil contre sa joue. Ils lui donnent un coup très fort en cherchant à le tuer. Nous nous penchons en avant les uns sur les autres. Et ils poussent certains des nôtres par terre et me poussent au milieu d'eux. ${ }^{27}$ Entre les militaires et les rebelles, je ne sais plus à quel saint me vouer. Puisqu'ils ne m'ont pas libéré comment pourrais-je m'enfuir d'entre ceux-ci ? ${ }^{28} \mathrm{C}$ 'est l'affaire des ancêtres, les ancêtres sont derrière moi. ${ }^{29}$ Voilà que nous sommes allés comme ça jusqu'à la base militaire, et ils nous ordonnent de rester là et à moi, il me demande de le conduire jusqu'en bas, au village là-bas. Le sucre qu'ils n'ont pas trouvé, les a fâchés. ${ }^{30} \mathrm{Il}$ trouve là l'occasion de s'enfuir et raconte comment il s'y est pris. Et c'est fini

\section{Récit de $\boldsymbol{G N}$}

${ }^{1}$ Commence par expliquer qu'il a tout fait, dès qu'il a entendu parler d'eux, pour éviter de se retrouver face aux rebelles. Mais il n'a pas pu les éviter. ${ }^{2}$ Resté au village dans la matinée et voulant partir pour les champs, des enfants lui demandent de l'aide : «papa voyez pour nous la base de cette maison qui nous dérange bien». Et je me penche et me baisse pour regarder, lorsque Y- : ${ }^{3}$ «oncle ! ceux que vous fuyiez, voyez, ils sont arrivés». ${ }^{4}$ Comme je cherche à me tourner et que je me dis «prends tes jambes à ton cou», ${ }^{5}$ il lève son fusil vers le haut. ${ }^{6}$ Aussitôt mes jambes se dérobent. Si je détale alors c'en est fini de moi. ${ }^{7} \mathrm{Il}$ est ensuite contraint de cueillir des mangues mûres. Pour cela il doit grimper dans le manguier. Mais il ne sait pas le faire. Deux jeunes présents y grimpent pour lui. ${ }^{8}$ Il les voit détruire des portes. ${ }^{9} \mathrm{Ce}$ jour là A- n'était pas bien. Ils disent : «C'est qui qu'est allongé ici ?». Le frère aîné A- : «je suis pas bien». Ils lui disent: «Ôte-toi de là ». ${ }^{10} \mathrm{Un}$ homme encore très jeune qui surgit comme P- qui est ici. Il m'appelle 'papa [en peul???]. Je fais oh de stupeur. Et il m'ordonne de me lever et de danser. Et moi je me lève bien sûr. ${ }^{11} \mathrm{Peu}$ après on va jusque chez $\mathrm{S}$ - là-bas. Comme je regarde j'en vois un autre qui se tient avec Y-, et ils parlent ensemble. Je m'adresse à Y-... J'appelle Y-. «Oh ! oh ! Je l'appelle, je ne vois pas qu'il est occupé à parler avec lui ?». «J'appelle Y- car c'est mon petit père», lui dis-je. ${ }^{12} \mathrm{Il}$ demande si je connais où il habite. Et Y- répond que oui, je sais où il habite. Alors que Y- me montre sa maison. Et Y- oui, c'est la maison là-bas. Alors que j'aille jusque chez Y- là-bas. ${ }^{13} \mathrm{~J}$ 'ai enfin trouvé le moyen de m'évaporer. Je vais à grands pas jusque chez G- là-bas. ${ }^{14} \mathrm{Il}$ est tenté par un plat de céphalophe roux et de boule abandonné devant une maison, mais il ne veut pas retarder sa fuite. Il s'enfonce sous les fleurs et va très loin. Voilà tout ce que j'ai vu de mes yeux.

\section{Récit de PD}

${ }^{1}$ A ce moment précis je n'avais pas de maison. Et je demeurai comme ça ${ }^{1}$ 'quand la situation devint dangereuse. J'étais en train de préparer de la boue pour faire des briques et je revenais d'avoir puisé de l'eau lorsque j'ai vu Z- qui courait. ${ }^{2}$ Est-ce qu'il est devenu fou ou est-il la proie d'un démon $?^{3}$ L'autre lui dit que ce sont les bandits. ${ }^{4}$ En voit un autre courir et se lamenter. ${ }^{5}$ Comme je revenais là, ils avaient déjà envahi le village. ${ }^{6} \mathrm{Et}$ je restais là. Moi : merde ! Les bandits dont le nom est célèbre je ne les ai encore pas vus. 
Je vais aller pour voir à quoi ils ressemblent. Je progresse en me cachant, les aperçois et ${ }^{7} \mathrm{j}$ 'ai réfléchi : merde ! Maman et les enfants sont tous aussi restés là. Je vais vraiment y retourner et j'irai voir. Si c'est la mort, je mourrai tout simplement avec eux, n'est-ce pas. Je vais et décide de revenir auprès des miens. ${ }^{8}$ Voilà que je reste là je baisse la tête un long moment lorsqu'un rebelle vient. ${ }^{9} \mathrm{Il}$ me dit qu'il est venu passer ici même et n'a vu aucun homme, d'où est-ce que je sors ? Il ne croit pas à mon histoire et pense que je suis allé caché quelque chose. ${ }^{10} \mathrm{Il}$ me demande alors de lui montrer les places des riches, il les trouvera et m'en donnera une part. ${ }^{11} \mathrm{Je}$ n'en connais pas et d'ailleurs les riches qui vivaient ici, les bandits les ont chassés, ils sont tous partis. Voilà qu'il m'ordonne de me lever. ${ }^{12}$ Et s'en prend à ma mère qu'il va questionner lui demandant pourquoi elle reste ainsi assise immobile? Insatisfait de sa réponse, il l'observe comme ça. ${ }^{13} \mathrm{Il}$ ajoute qu'elle et son enfant ils ne lui plaisent pas. ${ }^{14} \mathrm{Il}$ s'en va en m'emmenant pour aller attraper des poules. ${ }^{15}$ En dépit de mes protestations [dot], ${ }^{16} \mathrm{il} \mathrm{m}$ 'oblige à attraper les poules que je dois apporter à lui qui reste ici. Il me conduit ensuite sous le manguier. ${ }^{17}$ Là ils ont attrapé le grand-père N-. Ils ont ainsi ramené beaucoup de gens. Ils ont attrapé un Mbororo. Ils l'ont mis chez A- et l'ont mis au milieu de nous là. ${ }^{18} \mathrm{Et}$ ils demandent : nous en vérité estce que nous ne le connaissons pas ? ${ }^{19}$ Nous non 'on ne le connait pas'. N'est-il pas un peul comme lui ? ${ }^{20}$ Et lui il va le tuer en effet ensuite grâce à vous les Gbaya, dit-il, une seule balle suffit pour vous qui êtes ici. Vous êtes condamnés à mort aussi. ${ }^{21} \mathrm{Ils}$ commencent à découper les poules sous ce manguier. Et il m'ordonne d'aller chercher de l'eau. ${ }^{22}$ Tandis que je suis parti chercher cette eau je ne sais pas qu'ils ont partagé à tous la cola qu'ils avaient trouvé dans cette maison, après mon départ. ${ }^{23} \mathrm{~A}$ mon retour, il me donne une cola qu'il m'oblige à mettre entière dans ma bouche. ${ }^{24}$ Maintenant il veut que le grand $\mathrm{J}$ - il se lève, que le grand Y- il se lève, que G- se lève, qu'ils ramassent ses bagages, puis il se lève et il fera le jugement de G-plus tard là-bas. Puis ils se regroupent et partent. ${ }^{25}$ Moi je me contente de rester là seulement. Je me lève, pars et retourne dans la brousse là-bas, il fait alors déjà sombre. Ce que j'ai bien vu c'est cela

\section{Récit de BM}

${ }^{1} \mathrm{Au}$ moment où les rebelles sont venus au village de Ndongué ici, ${ }^{1}$ j'étais là aussi. Blessé au ventre je ne pouvais envisager de fuir et ai donc décidé de rester devant chez moi avec un jeune, également handicapé. Vers $9 \mathrm{~h}$ on a vu en haut du village là-bas ${ }^{2}$ le père $\mathrm{M}$ - qui courait vraiment, il partait en courant. «Oh ! Qu'est-ce qu'il y a qui le fait partir ici ?» Nous avons alors entendu dire 'les rebelles, les rebelles, les rebelles'. ${ }^{3}$ Tous les gens se sont mis à fuir. ${ }^{4}$ Alors un Mbororo qui a été vu et pourchassé est entré dans cette maison où son poursuivant a mis le feu mais il a réussi à s'enfuir. ${ }^{3}$ ' La fouille de la maison d'A-, beaucoup ont fui dans la savane. ${ }^{5}$ Moi tandis que je reste assis avec ma plaie, je vois qu'ils sortent la moto de l'aîné A-. Il est contraint à la pousser car il n'arrive pas à démarrer. ${ }^{6}$ Pendant ce périple il me demande c'est quoi cette maison-là. Je réponds vois ! le propriétaire est parti à Carnot là-bas. ${ }^{7}$ Trajet dans le village, passe près d'un très gros avocatier. Pour finir, ${ }^{8} 1$ 'interroge sur son bandage. ${ }^{9}$ Ensuite ils entreprennent de ramasser les poules. ${ }^{9}$ 'Puis nous tuons les poules et donc nous passons les poules à la flamme pour 
qu'ils le mangent, lui proposant de manger tout ce qu'ils ne mangent pas. ${ }^{10}$ Certains de ceux qui étaient aux champs ont entendu ce qui se passe au village et pensent qu'ils m'ont sans doute eu. ${ }^{11}$ Puis ils me demandent si D- qui se trouve à côté de moi et avait les cheveux tressés, était une fille. ${ }^{12}$ Enfin les radios, les autres les boubous, les vêtements des haoussa, toutes ces choses qui restent là, il me dit de les prendre pour moi puisque j'ai cuit pour lui. C'est leur première incursion.

\section{Récit de MG}

${ }^{1}$ De ce qui nous est arrivé en pleine brousse là-bas à la Wei là-bas je veux témoigner. ${ }^{2}$ A ce moment-là je ne voulais pas en parler parce que cette situation, contient des choses qui ne vont pas. Je vais cependant le raconter. ${ }^{3}$ Nous sommes partis, nous nous y sommes mis [au gravier pour chercher de l'or]. Le lendemain nous y retournons pour le laver encore une fois. A ce moment-là nous sommes allés prendre du manioc au village. ${ }^{4} \mathrm{Or}$ juste après que nous sommes partis comme ça ils sont arrivés au village. Ils ne nous ont pas rencontrés. ${ }^{5}$ Ils sont allés nous trouver là où nous étions allés pour laver le gravier. ${ }^{6}$ Nous avons aussitôt sursauté comme un seul homme. ${ }^{7}$ «Ne bougez pas oui, ne vous enfuyez pas. Si nous fuyons il nous frappera». ${ }^{8} \mathrm{Ils}$ nous fouillent. Quand ils trouvent quelque chose, et ils s'en emparent. ${ }^{9}$ Puis ils veulent se reposer et ils nous ordonnent de nous asseoir, puis ils se mettent à servir des cafés. ${ }^{10}$ Puis comme mon frère Ganimba s'est levé, ils lui demandent pourquoi, puis l'un lui donne un coup de pied dans le foie, puis il se tourne et prend la crosse du fusil et il la lance contre son foie. Il a reçu un grand choc déjà. ${ }^{11} \mathrm{Au}$ moment du départ, alors qu'il fait déjà bien sombre nous cherchons un endroit pour dormir. ${ }^{12}$ Après qu'ils ont frappé Ganimba il est venu me mettre une corde autour de mon cou et l'ont laissé comme mort. ${ }^{13}$ Le chef dit «oh! ça suffit oh! vois! je suis mort» et ils me laissent tomber par terre. Voilà que mon corps est tout mou, la vie est encore là c'est plus fort. Ca dure, ça dure une heure, puis ensuite je me remets à respirer lentement. ${ }^{14}$ Puis le Caméléon déguisé se décolle et donc je vais tomber dans l'eau, et je suis allongé sous l'eau comme ça, longtemps, puis alors mon corps revient et je vois que je m'avance donc pour m'asseoir par terre. ${ }^{15} \mathrm{Ils}$ sont partis. Nous sommes rentrés au village avec le mort. ${ }^{16}$ Une telle espèce de personne ce n'est vraiment pas supportable. 
